\title{
A desconexão do conhecimento químico com o conhecimento pedagógico para o ensino de Química
}

\author{
The disconnection of chemical knowledge with pedagogical knowledge for Chemistry teaching \\ La desconexión del conocimiento químico con el conocimiento pedagógico para la enseñanza de la
}

Química

Recebido: 13/10/2021 | Revisado: 23/10/2021 | Aceito: 31/10/2021 | Publicado: 01/11/2021

\author{
Susel Taís Soares \\ ORCID: https://orcid.org/0000-0003-4681-968X \\ Instituto Federal de Mato Grosso, Brasil \\ E-mail: susel.soares@ifmt.edu.br \\ Marcela Marques \\ ORCID: https://orcid.org/0000-0001-5537-0461 \\ Secretaria de Estado de Educação de Mato Grosso, Brasil \\ E-mail:m.marquesbio@gmail.com \\ Leandro Carbo \\ ORCID: https://orcid.org/0000-0001-5514-7040 \\ Instituto Federal de Mato Grosso, Brasil \\ E-mail: leandro.carbo@ifmt.edu.br
}

\begin{abstract}
Resumo
A literatura aponta a falta de conhecimento pedagógico por parte de professores que possuem bom domínio do conteúdo como uma das grandes problemáticas no ensino de Química. No entanto a abordagem deste aspecto se baseia principalmente em impressões pessoais dos atores das pesquisas. Para que uma pesquisa constate de modo mais empírico esta desconexão entre o conteúdo e a didática no ensino da Química faz-se necessário que os conhecimentos mobilizados durante a prática docente sejam evidenciados, descritos e identificados como conhecimentos Químicos ou Pedagógicos. Dessa forma, o objetivo desta pesquisa foi averiguar a hipótese apresentada na literatura sobre a falta de conexão do Conhecimento Químico com o Conhecimento Pedagógico do Conteúdo, utilizando o modelo Conhecimento Especializado de Professores de Química analisando episódios de ensino, seguido de tratamento matemático dos dados. Conforme apontado na literatura, tal desconexão entre os conhecimentos foi comprovada, através da discrepância significativa entre estes conhecimentos evidenciados em episódios de ensino de química.
\end{abstract}

Palavras-chave: MTSK; Ensino de Química; Conhecimento químico; CTSK.

\begin{abstract}
Literature points out the lack of pedagogical knowledge on the part of teachers who have good mastery of the content as one of the major problems in teaching Chemistry. However, the approach to this aspect is mainly based on personal impressions of the research actors. In order for a research to confirm in a more empirical way this disconnection between the content and the didactics in the teaching of Chemistry, it is necessary that the knowledge mobilized during the teaching practice is evidenced, described and identified as Chemistry or Pedagogical knowledge. Thus, the objective of this research was to investigate the hypothesis presented in the literature about the disconnection of Chemistry Knowledge from Pedagogical Content Knowledge, through the application of the Chemistry Teacher's Specialized Knowledge of model, analyzing teaching episodes, followed by mathematical treatment of the data. As pointed out in the literature, such a disconnect between knowledge was proven through the significant discrepancy between this knowledge evidenced in episodes of teaching chemistry.
\end{abstract}

Keywords: MTSK; Chemistry teaching; Chemistry knowledge; CTSK.

\section{Resumen}

La literatura señala la falta de conocimientos pedagógicos por parte de los docentes que tienen un buen dominio de los contenidos como uno de los mayores problemas en la enseñanza de la Química. Sin embargo, el enfoque de este aspecto se basa principalmente en las impresiones personales de los actores de la investigación. Para que una investigación confirme de manera más empírica esta desconexión entre el contenido y la didáctica en la enseñanza de la Química, es necesario que el conocimiento movilizado durante la práctica docente se evidencie, describa e identifique como conocimiento químico o pedagógico. Así, el objetivo de esta investigación fue indagar en la hipótesis presentada en la literatura sobre la desconexión del Conocimiento Químico del Conocimiento del contenido 
pedagógico, mediante la aplicación del modelo de Conocimientos Especializados de Profesores de Química, analizando episodios de enseñanza, seguido del tratamiento matemático de los datos. Como se señala en la literatura, tal desconexión entre conocimientos se demostró a través de la significativa discrepancia entre estos conocimientos evidenciada en episodios de enseñanza de la química.

Palabras clave: MTSK; Enseñanza de la Química; Conocimientos químicos; CTSK.

\section{Introdução}

A educação, embora tenha se consolidado a partir do século XVII, com a criação das instituições de ensino, ainda nos dias atuais permanece em processo de transformação devido aos problemas que enfrenta em seu cotidiano. Entretanto estes problemas não podem ser retratados como algo específico da educação, uma vez que englobam aspectos além dos educacionais, como sociais, financeiros, culturais e, até mesmo, históricos (Coimbra, 1989).

A realidade da educação brasileira é especialmente precária, pois além dos obstáculos mencionados há problemas como a falta de profissionais adequadamente capacitados em muitas áreas (Brasil, 2017), o fato dos cursos de licenciatura não serem valorizados (Oliveira \& Rezende, 2011), assim como a falta de reconhecimento profissional e, consequentemente, a baixa remuneração dos docentes (Fuentes, 2014; Ferreira, 2018).

A disciplina de Química é uma disciplina consideravelmente nova, pois os conteúdos relativos à Química eram desenvolvidos como parte do ensino de Física, inclusive não havia no país cursos para profissionais da área química, algo que somente em 1919 que houve a implantação do primeiro curso de nível superior na área (Silva \& Schnetzler, 2005; Lima, 2018). Apesar de ser uma disciplina contemporânea, ela também apresenta problemas como todas as outras do currículo educacional, dentre estes problemas a literatura relata a complexidade da disciplina (Quadros et al., 2011), formação de qualidade questionável de profissionais da educação devido à falta de conexão dos conhecimentos, da Química com a parte pedagógica em diferentes contextos (Lopes, 1997; Garcia, 2009; Oliveira \& Rezende, 2011).

Nas últimas quatro décadas uma série de pesquisas foram desenvolvidas a partir do modelo proposto por Shulman (1986), denominado Pedagogical Content Knowledge (PCK), Conhecimento Pedagógico do Conteúdo, dentre todas as áreas de conhecimento a matemática foi a área de maior destaque com pesquisas a partir do PCK (Goes, 2014).

Considerando o destaque dos estudos na área da matemática, no ano de 2008 teve-se a proposta de um novo modelo teórico que foi o Mathematics Knowledge for Teaching (MKT), Conhecimento Matemático para o Ensino (Ball; Thames \& Phelps, 2008) que foi um modelo importante para a área, entretanto foram identificadas algumas lacunas que ocasionou a proposta de um novo modelo em 2014, que foi o Mathematics Teacher's Specialized Knowledge (MTSK), Conhecimento Especializado de Professores de Matemática (Carrillo et al., 2014) e que devido à relevância e repercussão do modelo, houve a proposta de transposição deste modelo especializado para a área da biologia, estudos iniciados em 2015 e que resultou em diversos trabalhos, sendo o mais atual que foi a tese defendida em 2021 (Luís; Monteiro \& Carrillo, 2015; Luís, 2021).

Enquanto isso no Brasil, alguns estudos vêm sendo realizados a partir do MTSK (Moriel Junior, 2014; Moriel Junior \& Wielewski, 2017; Vasco; Moriel Junior \& Contreras, 2017), não somente na área da matemática, mas inclusive com a transposição para as distintas áreas das ciências naturais: Física, Química e Biologia (Lima et al., 2017; Lima, 2018; Soares, 2019; Marques, 2020).

Este modelo teórico possibilita descrever o Conhecimento Especializado de Professores de Matemática, contemplando a conexão entre os conhecimentos, Conhecimento Matemático e o Conhecimento Pedagógico do Conteúdo matemático, uma característica importante para o processo de ensino e de aprendizagem e sendo a base da proposta do modelo Conhecimento Especializado de Professores de Química - CTSK.

Deste modo, baseando-se nas informações da literatura de relatos sobre a falta de conexão dos Conhecimentos, da Química com o Pedagógico e nos modelos teóricos desenvolvidos no decorrer das últimas quatro décadas, esta pesquisa teve 
por objetivo aplicar o modelo CTSK para averiguar a problemática relatada na literatura analisando dois episódios de ensino, este trabalho trata-se de um recorte da dissertação de mestrado da primeira autora.

\section{Conhecimento Especializado de Professores de Química}

No ano de 2019, foi proposto o modelo Conhecimento Especializado de Professores de Química, Chemistry Teacher's Specialized Knowledge - CTSK (Soares, 2019) capaz de descrever e caracterizar os Conhecimento Especializado de Professores de Química, desde então este modelo tem sido aplicado em algumas pesquisas do ensino de química (Floriano, 2021; Martins, 2021; Martins; Carbo \& Soares, 2021). A pesquisa que possibilitou a proposta do modelo CTSK foi desenvolvida no Brasil, como projeto de mestrado desenvolvido no Grupo de Pesquisa TSK do Instituto Federal de Educação, Ciência e Tecnologia de Mato Grosso, onde tem-se o desenvolvimento de pesquisas na área da matemática, da física, da química e da biologia, a partir do MTSK (Moriel Junior, 2014; Moriel Junior \& Wielewski, 2017; Vasco; Moriel Junior \& Contreras, 2017; Lima et al., 2017; Lima, 2018; Soares, 2019; Soares et al., 2020; Marques, 2020).

O CTSK possui dois domínios, sendo eles o Conhecimento Químico e o Conhecimento Pedagógico do Conteúdo, na qual cada um destes domínios possuem três subdomínios, conforme é possível visualizar na Figura 1 e no Quadro 1 (Soares, 2019).

Figura 1 - Modelo do Conhecimento Especializado de Professores de Química.

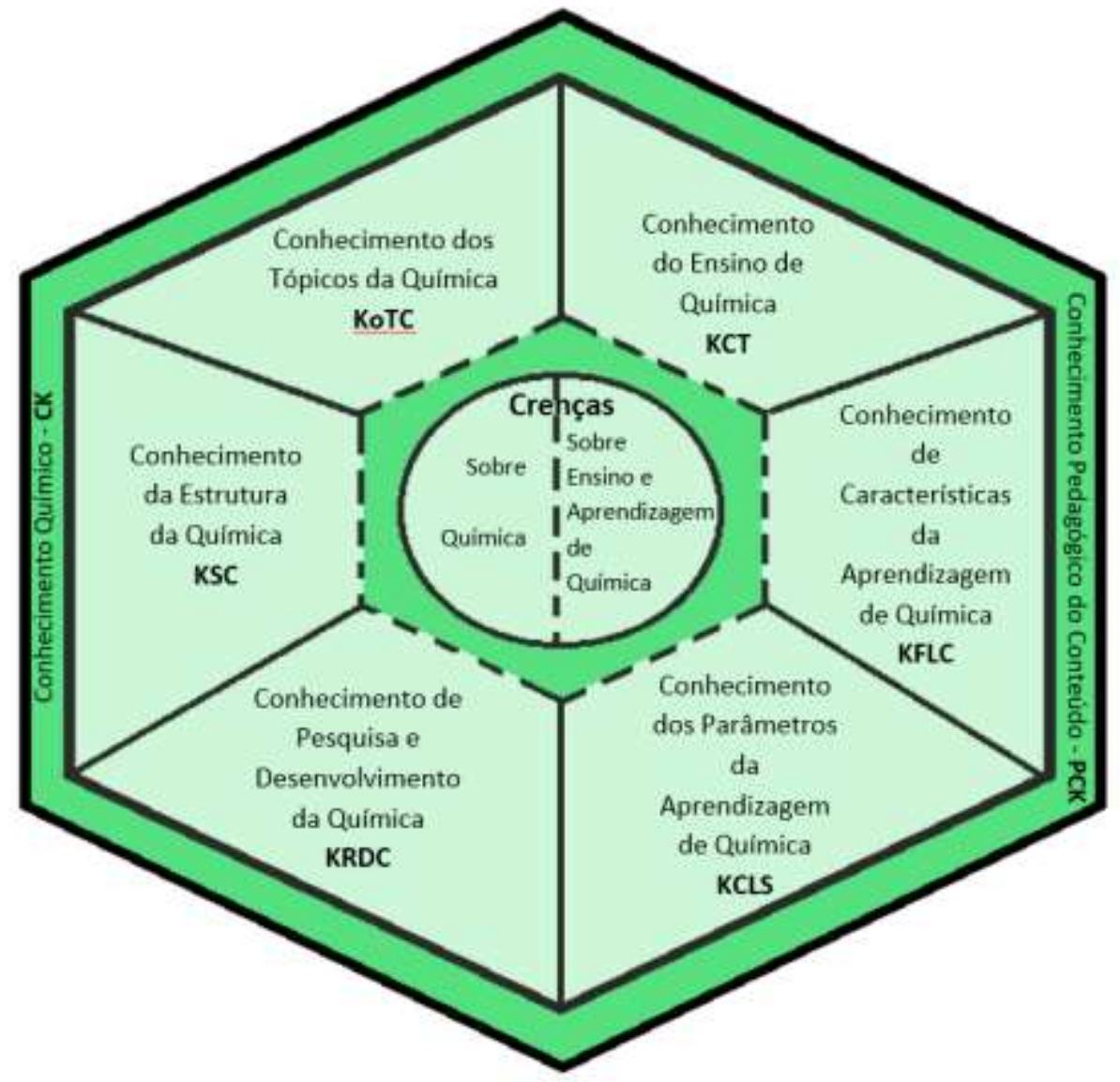

Fonte: Soares (2019, adaptado pelos autores). 
Quadro 1 - Descrição dos subdomínios do CTSK.

\begin{tabular}{|c|c|c|}
\hline \multicolumn{3}{|r|}{ Domínios } \\
\hline Sigla & Subdomínios & Descrição dos Conhecimentos referentes aos Subdomínios \\
\hline \multicolumn{3}{|c|}{ CK - Chemistry Knowledge (Conhecimento Químico) } \\
\hline 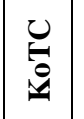 & $\begin{array}{l}\text { Knowledge of Topics of Chemistry } \\
\text { (Conhecimento dos Tópicos da } \\
\text { Química) }\end{array}$ & $\begin{array}{l}\text { Modelos químicos, teorias, leis, conceitos, definições, fundamentos, fórmulas } \\
\text { químicas, gráficos, representações, aplicações, experimentação, conhecimento } \\
\text { interdisciplinar e história da química. }\end{array}$ \\
\hline \begin{tabular}{l} 
Un \\
\multirow{1}{*}{}
\end{tabular} & $\begin{array}{l}\text { Knowledge of the Structure of } \\
\text { Chemistry } \\
\text { (Conhecimento da Estrutura da } \\
\text { Química) }\end{array}$ & $\begin{array}{l}\text { Estrutura da química e suas conexões, vinculando diferentes conceitos. Abrange } \\
\text { a relação dos conhecimentos transmitidos e os que serão ensinados, porém em } \\
\text { diferentes ocasiões, podendo ocorrer estas conexões por: complexidade, } \\
\text { simplificação, conexão transversal, conexão auxiliar, conexão experimental, } \\
\text { aplicabilidade e representação. }\end{array}$ \\
\hline 㫕 & $\begin{array}{l}\text { Knowledge of Research and } \\
\text { Development of Chemistry } \\
\text { (Conhecimento de Pesquisa e } \\
\text { Desenvolvimento da Química) }\end{array}$ & $\begin{array}{l}\text { Conhecimentos referentes ao desenvolvimento investigativo da química para } \\
\text { gerar novos conhecimentos referentes à química pura. Englobando } \\
\text { conhecimentos relativos a deduções químicas; desenvolvimento de } \\
\text { metodologias teóricas/ experimentais relativas à química pura; pesquisa } \\
\text { investigativa e desenvolvimento argumentativo com exemplos e } \\
\text { contraexemplos, seja teóricos e/ou experimentais; pesquisa relativa ao } \\
\text { levantamento de hipóteses, e; desenvolvimento de processos. }\end{array}$ \\
\hline \multicolumn{3}{|c|}{ PCK - Pedagogical Content Knowledge (Conhecimento Pedagógico do Conteúdo) } \\
\hline 论 & $\begin{array}{l}\text { Knowledge of Chemistry Teaching } \\
\text { (Conhecimento do Ensino de } \\
\text { Química) }\end{array}$ & $\begin{array}{l}\text { Conhecimento do docente mediante a potencialidade de determinada atividade } \\
\text { perante o processo de aprendizagem; os recursos materiais, laboratoriais e } \\
\text { virtuais, ou seja, desde materiais básicos à todas as áreas como data show, lousa } \\
\text { digital, livros, revistas, jornais, ou materiais de uso específico da química como } \\
\text { tabela periódica, modelo molecular 3D, ou materiais laboratoriais como } \\
\text { vidrarias e equipamentos como pHmetro, capela, estufa, entre outros, até } \\
\text { recursos virtuais como softwares tanto desenvolvidos para química ou não, } \\
\text { desde que aplicáveis, o uso de aplicativos, internet ou vídeos. }\end{array}$ \\
\hline ב্] & $\begin{array}{l}\text { Knowledge of Features of } \\
\text { Learning Chemistry } \\
\text { (Conhecimento das Características } \\
\text { de Aprendizagem da Química) }\end{array}$ & $\begin{array}{l}\text { Ao processo de assimilação, erros e dificuldades, de modo que o conteúdo } \\
\text { químico seja o objeto de aprendizagem e não o aluno, porém faz-se necessário } \\
\text { considerar a relação aluno-objeto, independentemente da particularidade deste } \\
\text { estudante. Este subdomínio considera a forma como o aluno aprende conteúdos } \\
\text { de Química, qual é o interesse e a expectativa deste aluno quanto à determinada } \\
\text { área da Química, o que pode ser considerado um elemento facilitador ou o que } \\
\text { pode imobilizar o conhecimento da química, ou em determinada área da } \\
\text { Química. }\end{array}$ \\
\hline$\underset{\mathcal{U}}{\tilde{U}}$ & $\begin{array}{l}\text { Knowledge of Chemistry Learning } \\
\text { Standards } \\
\text { (Conhecimento dos Parâmetros da } \\
\text { Aprendizagem de Química) }\end{array}$ & $\begin{array}{l}\text { Aos parâmetros curriculares, a sequência dos conteúdos conforme o nível } \\
\text { escolar, considerando a interdisciplinaridade da química, a expectativa do } \\
\text { professor com relação a determinada aprendizagem em determinado nível } \\
\text { escolar e a meta quanto ao desenvolvimento de determinado conteúdo químico. }\end{array}$ \\
\hline
\end{tabular}

Fonte: Soares (2019).

\section{Metodologia}

Trata-se de uma pesquisa de cunho qualitativo (Bogdan \& Biklen, 1994), a coleta de dados foi através da análise de dois episódios de ensino aleatórios, mediante a utilização do Relatório da Experiência Profissional Pedagógica, Pedagogical and Professional-experience Repertoire - PaP-eR, que documenta um episódio de ensino, a partir de uma prática real de um conteúdo específico de uma área (Loughran et al., 2001). Essa ferramenta já foi utilizada por pesquisadores para documentar o PCK (Goes, 2014) e como metodologia aplicada no ensino de física (Lima, 2018), de biologia (Marques \& Moriel Junior 2019; Marques \& Moriel Junior, 2020; Marques, 2020; Marques et al., 2021) e também de química (Soares, 2019; Soares et al., 
Para a seleção dos episódios de ensino alguns critérios foram obedecidos, tais como: ser baseado em uma prática real de ensino de Química; possibilitar a reconstrução do episódio de ensino; apresentar descrições detalhadas do cenário de ensino; e abordar o aspecto experimental do ensino de Química.

Após a identificação de cada conhecimento evidenciado foram atribuídos respectivos comentários de análise; para isso utilizou-se a seguinte regra: "conhecimento de (...) que consiste em...”, conforme metodologia aplicada por Lima (2018). A finalidade de utilizar estes comentários foi assegurar que a classificação do tipo de conhecimento atribuída foi corretamente.

A identificação dos conhecimentos foi atribuída obedecendo a seguinte regra: primeiro adicionando a letra "e" indicando o episódio de ensino que estava em análise, seguido do número atribuído a este episódio, conforme denominação supracitada; depois foi indicado qual o tipo de conhecimento evidenciado, Conhecimento Químico ou Conhecimento Pedagógico, aplicando o modelo do Conhecimento Especializado de Professores de Química - CTSK como ferramenta analítica, atribuindo a ordem de identificação no respectivo episódio de ensino, representada com dois algarismos arábicos, conforme explicado na Figura 2.

Figura 2 - Regra para identificação do conhecimento.

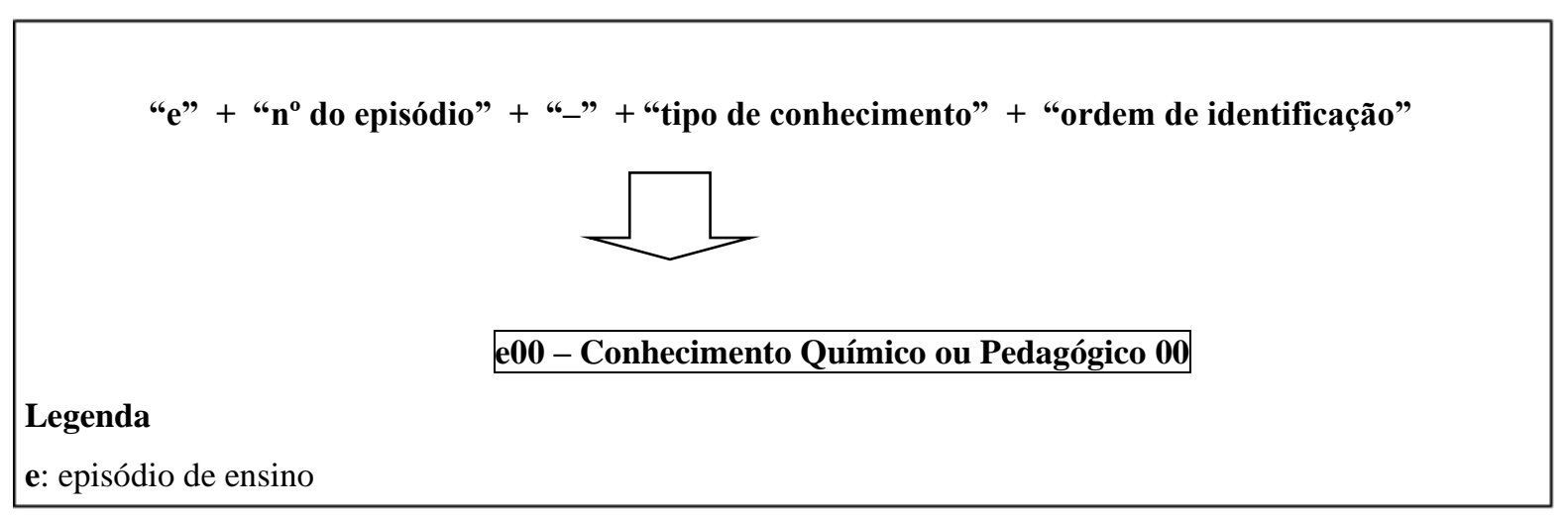

Fonte: Soares (2019).

O tratamento dos resultados foi realizado através da interpretação e confronto sistemático com base nos argumentos de Bardin (1995).

\section{Resultados e Discussão}

Foram selecionados 2 episódios de ensino em diferentes áreas da Química, sendo o episódio 01, denominado e01, com conhecimentos nas áreas de química geral, inorgânica, físico-química, orgânica e química ambiental e o episódio 02, denominado e02, específico da área de química orgânica. Os quadros 2 e 3 apresentam as informações dos PaP-eR analisados nesta pesquisa. 
Quadro 2 - Primeiro episódio de ensino selecionado para análise.

\begin{tabular}{|l|}
\hline \multicolumn{1}{|c|}{ EPISÓDIO $1 \rightarrow$ e01 } \\
\hline Cavalcanti, J. A.; Freitas, J. C. R.; Melo, A. C. N.; Freitas Filho, J. R. (2010) Agrotóxicos: Uma Temática para \\
o Ensino de Química. Química Nova na Escola, v. 32, nº 1, p. 31-36. \\
Relato de episódio de ensino \\
Temática: Agrotóxicos. \\
Séries do Ensino Médio: $1^{\text {a }} 2^{\text {a e } 3^{\text {a }}}$ \\
Áreas de abrangência: Química Geral, Inorgânica, Físico-química, Orgânica e Química Ambiental \\
Contextualiza: Substâncias, Misturas, Tabela Periódica, Noção de Química Ambiental, Funções \\
Químicas, Soluções, Estudo do carbono e Funções Orgânicas. \\
\hline
\end{tabular}

Fonte: Soares (2019).

Quadro 3 - Segundo episódio de ensino selecionado para análise.

\begin{tabular}{l} 
EPISÓDIO $2 \rightarrow$ e02 \\
\hline Pazinato, M. S.; Braibante, H. T. S.; Braibante, M. E. F.; Trevisan, M. C.; Silva, G. S. (2012) Uma \\
Abordagem Diferenciada para o Ensino de Funções Orgânicas através da Temática Medicamentos. Química \\
Nova na Escola, v. 34, $\mathrm{n}^{\circ}$ 1, p. 21-25. \\
Relato de episódio de ensino \\
Temática: Medicamentos. \\
Série do Ensino Médio: $3^{\text {a }}$ \\
Áreas de abrangência: Química Orgânica \\
Contextualiza: Funções Orgânicas
\end{tabular}

Fonte: Soares (2019).

No total foram evidenciados 80 conhecimentos. Apesar do e01 contextualizar vários conteúdos, apresentou evidências de poucos conhecimentos, comparado ao e02, sendo identificados 26 e 54 conhecimentos em cada episódio respectivamente.

Em uma visão geral dos resultados obtidos, $28 \%$ referem-se ao Conhecimento Pedagógico, como partes integrantes destes resultados estão os conhecimentos relativos ao ensino de química, estratégia, características de aprendizagem e conhecimento do currículo da química e suas respectivas divisões, conforme o nível de ensino.

Os outros $73 \%$ referem-se ao Conhecimento Químico, dentre os conhecimentos identificados 69\% correspondiam ao Conhecimento dos Conteúdos Químicos, desde reação, representação, nomenclatura até aplicação. Os outros $4 \%$ estavam relacionados à conexão entre conteúdos químicos de diferentes áreas da Química.

$\mathrm{Na}$ análise individual dos episódios de ensino, em e01, foram evidenciados 26 conhecimentos no total, sendo 7 referentes ao Conhecimento Químico e 19 referentes ao Conhecimento Pedagógico. Este primeiro resultado apontou uma desconexão de conhecimentos significativa, uma vez que 73\% dos conhecimentos correspondem ao Pedagógico. O Quadro 4 apresenta um exemplo de cada Conhecimento, químico e Pedagógico, evidenciados no e01. 
Quadro 4 - Exemplos de conhecimentos evidenciados no e01.

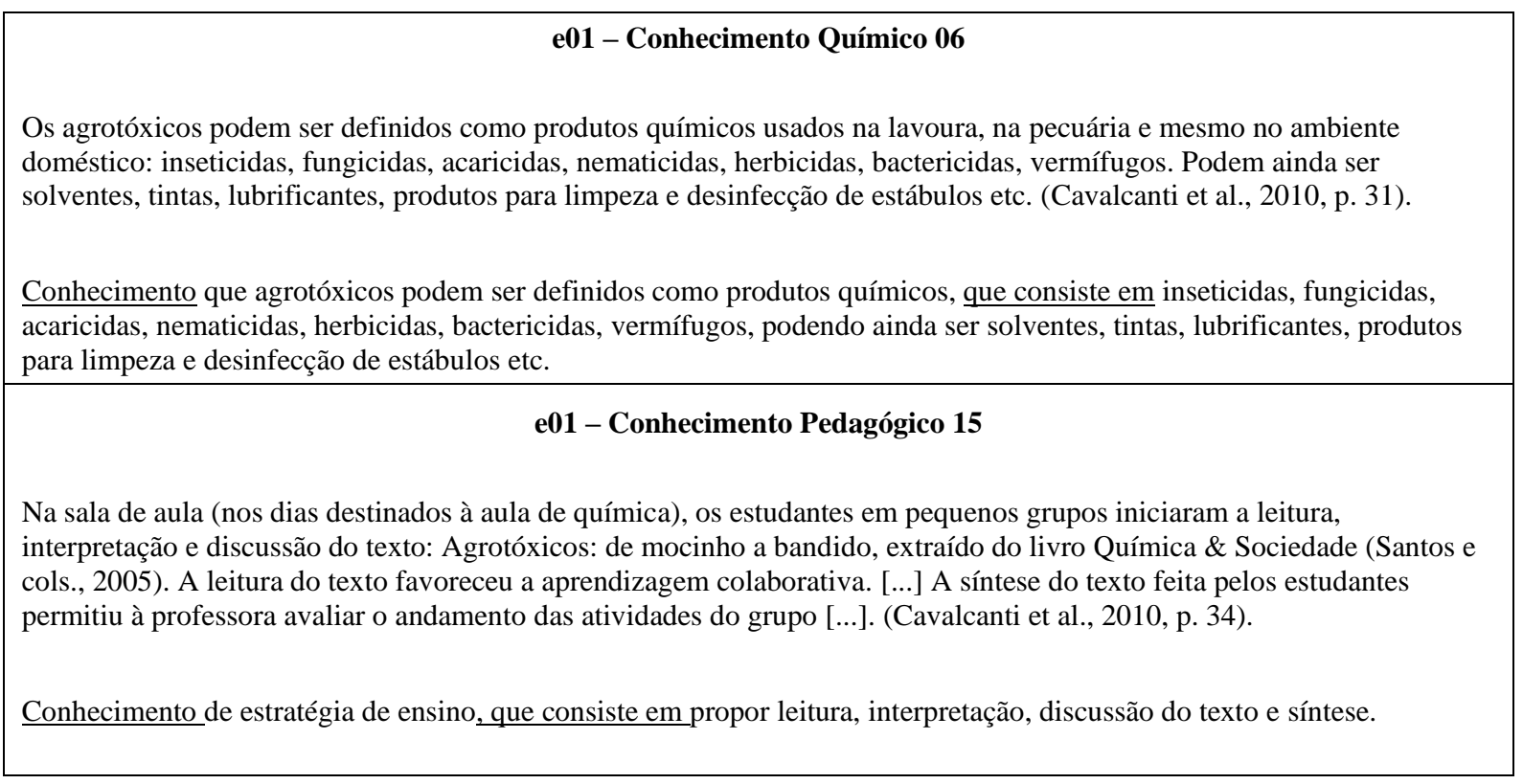

Fonte: Autores.

Desde modo, fez-se o tratamento de dados da análise do episódio de ensino e02, na qual foram evidenciados 54 conhecimentos no total, sendo 51 referentes ao Conhecimento Químico e 3 referentes ao Conhecimento Pedagógico. Um resultado com uma característica oposta em relação ao e01, uma vez que apresentou mais evidências de Conhecimentos Químicos do que de Conhecimentos Pedagógicos, entretanto houve uma discrepância ainda mais considerável de conhecimentos, haja vista que $94 \%$ referiam-se ao Conhecimento Químico. O Quadro 5 apresenta um exemplo de cada conhecimento, Químico e Pedagógico, evidenciados no e02 e a Figura 3 apresenta os resultados obtidos em geral e em cada relato de episódio de ensino analisado. 
Quadro 5 - Exemplos de conhecimentos evidenciados no e02.

\section{e02 - Conhecimento Químico 41}

Os fenóis, ao reagirem com cloreto férrico $\left(\mathrm{FeCl}_{3}\right)$, formam complexos coloridos, sendo esta uma das reações que identificam esses compostos [...]. A coloração do complexo formado varia do azul ao vermelho, dependendo do solvente. Essa reação pode ocorrer em água, metanol ou diclorometano. (Pazinato et al., 2012, p. 23).

Conhece as implicações na escolha do solvente para reação dos fenóis com cloreto férrico que consiste na diferença de cores, variando do azul ao vermelho.

\section{e02 - Conhecimento Pedagógico 52}

[...] atividade experimental de identificação de grupos funcionais, utilizando medicamentos como reagentes (Pazinato et al., 2012, p. 22)

[...] $3^{\text {a }}$ Etapa- Atividade experimental: As abordagens experimentais auxiliam na construção do conhecimento científico, pois a organização do conhecimento ocorre preferencialmente nos entremeios da investigação. A atividade experimental apresentada neste trabalho foi desenvolvida com graduandos do curso de Química Licenciatura, no espaço Ciência Viva - UFSM, e teve por objetivo propor uma maneira diferenciada, da que é desenvolvida pelos professores e proposta pelos livros Pedagógicos, na abordagem do conteúdo de grupos funcionais por intermédio da utilização da temática medicamentos. Essa atividade experimental os utiliza como amostras reais para a identificação dos grupos funcionais presentes nas estruturas químicas dos seus princípios ativos. (Ibid., p. 24).

Conhecimento de estratégia de ensino que consiste na atividade experimental de identificação de grupos funcionais, utilizando medicamentos como reagentes que consiste na descrição feita no trecho " $3^{\mathrm{a}}$ etapa Atividade Experimental" do item metodologia desenvolvida do artigo.

Fonte: Soares (2019).

Figura 3 - Porcentagem dos conhecimentos evidenciados.

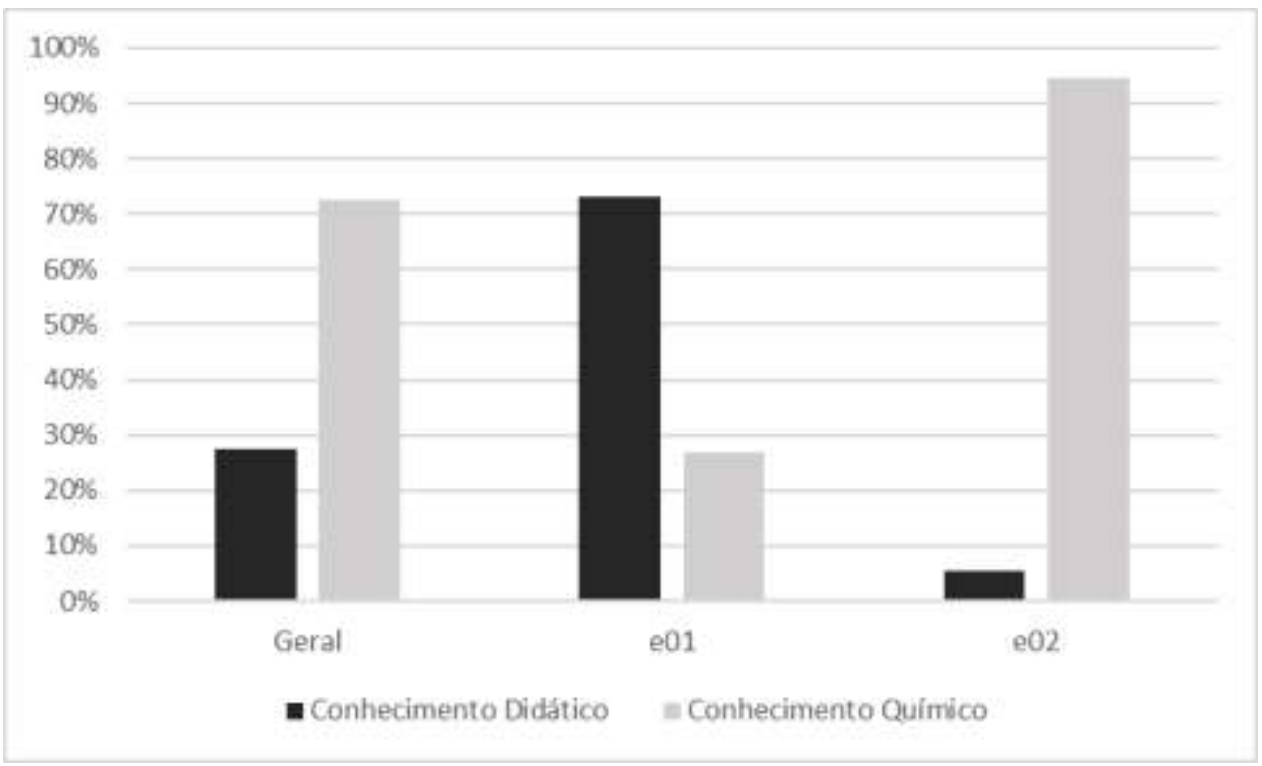

Fonte: Autores.

Dentre as problemáticas evidenciadas na literatura a falta da conexão do Conhecimento Químico com o Conhecimento Pedagógico do Conteúdo de química é a uma das mais frequentes, devido a uma falha nos cursos de licenciatura. Muito embora a literatura apresente pesquisas que possibilitam identificar os problemas e propor técnicas e 
experimentos para ensinar determinado conteúdo, estas propostas não conectam as informações dos conhecimentos químicos e pedagógicos, ora valorizam um, ora valorizam outro conhecimento, ou seja, esta falta de conexão provoca problemas no processo de ensino e aprendizagem da Química (Garcia, 2009; Oliveira \& Rezende, 2011). Tal fato foi comprovado matematicamente através dos resultados provenientes do e02, quando observa-se a predominância significativa do conhecimento químico em um relato de episódio de ensino, com mais de $94 \%$ das evidências, ou seja, uma desvalorização do Conhecimento Pedagógico relativo à este determinado conteúdo.

Entretanto, o contrário também foi evidenciado no e01, na qual o foco foi referente a parte Pedagógica, remetendo ao que a literatura questionou quanto a qualidade da formação docente (Garcia, 2009). Na pesquisa desenvolvida por Oliveira \& Rezende (2011) teve-se um relato de um estudante do curso de licenciatura que apontou a desarticulação existente na área de formação docente de química quanto as disciplinas pedagógicas e o conteúdo específico dentro de uma universidade brasileira, na qual os autores alegam que há um problema significante na formação de professores de Química.

\section{Considerações Finais}

Os resultados obtidos atenderam ao objetivo desta pesquisa, uma vez que possibilitou fomentar a hipótese de que realmente há uma desconexão significativa de Conhecimentos Químicos e Conhecimentos Pedagógicos por parte dos professores, assim como apontado na literatura.

Analisando os dados gerais, verifica-se uma predominância dos conhecimentos químicos dentre os conhecimentos evidenciados, um fator preocupante para o processo de ensino aprendizagem para a área da química. Entretanto, uma vez comprovada através de dados matemáticos que realmente existe esta discrepância dos dois aspectos do conhecimento, o Químico e o Pedagógico, é possível os docentes iniciarem trabalhos com intuito de minimizar esta desconexão, podendo vir a influenciar positivamente no processo de ensino-aprendizagem na área. Assim como, é possível adequar projetos pedagógicos de cursos de licenciatura na área para que os futuros profissionais licenciados em química possam atender a demanda existe, relacionada à esta problemática evidenciada. Inclusive, sugere-se pesquisas na área que possam contribuir nestas duas frentes supracitadas, para integração destes conhecimentos e minimizar esta problemática no cotidiano escolar.

\section{Referências}

Ball, D. L., Thames, M. H. \& Phelps, G. (2008) Content Knowledge for Teaching: What Makes It Special? Journal of teacher education, SAGE, 59(5), 389407.

Bardin, L.(2016) Análise de Conteúdo. Edições 70. 281p.

Bogdan, R. \& Biklen, S. (1994) Investigação qualitativa em Educação: fundamentos, métodos e técnicas. Porto Editora. 47-51

Brasil. (2017) Ministério da Educação. Instituto Nacional de Estudos e Pesquisas Educacionais Anísio Teixeira - INEP (Comp.). Censo escolar da educação básica 2016: Notas estatísticas. Brasília. 29 p.

Carrillo, J., Avila, D. I. E., Mora, D. V., Medrano, E. F. (2014) Un marco teórico para el conocimiento especializado del profesor de matemáticas. Huelva, Espanha: Universidad de Huelva Publicaciones.

Cavalcanti, J. A., Freitas, J. C. R., Melo, A. C. N., Freitas Filho, J. R. (2010) Agrotóxicos: Uma Temática para o Ensino de Química. Química Nova na Escola, 32(1), 31-36.

Coimbra, C. M. B. (1989) As funções da instituição escolar: análise e reflexões. Psicologia: ciência e profissão, 9(3), 14-16.

Ferreira, P. (2018) Salário mínimo pago ao professor no Brasil é um dos piores do mundo. O Globo Digital, 11/09/2018.

Floriano, L. S. (2021) Conhecimento Especializado de professores de química (CTSK): um estudo de caso do ensino de termoquímica nas práticas de dois professores de Cuiabá - MT. Dissertação (Mestrado em Ensino) - Programa de Pós-Graduação Stricto Sensu em Ensino, Instituto Federal de Educação, Ciência e Tecnologia de Mato Grosso - IFMT, Cuiabá. 116f.

Fuentes, A. (2014) Salário dos professores brasileiros está entre os piores do mundo. Veja, 10/09/2014. 
Garcia, I. T. S. (2009) Implantação das Diretrizes Curriculares Nacionais para Formação de Professores de Química em uma Instituição Federal de Ensino Superior: Desafios e Perspectivas. Química Nova, SBQ, 32(8), 2218-2224.

Goes, Luciane F. (2014) Conhecimento Pedagógico do conteúdo: estado da arte no campo da educação e no ensino de química. Dissertação (Mestrado em Ensino de Química) - Universidade de São Paulo - USP. 155 p.

Lima, S. S. (2018) Conhecimento especializado de professores de física: uma proposta de modelo teórico. Dissertação (Mestrado em Ensino) - Programa de Pós-Graduação Stricto Sensu em Ensino, Instituto Federal de Educação, Ciência e Tecnologia de Mato Grosso - IFMT, Cuiabá. 293 f.

Lima, S. S., Costa, L. D., Soares, S. T. C., Silva Filho, V. P., Moriel Junior, J. G., Mello, G. J. (2017) Análise de PaP-eRs como primeira aproximação metodológica para configurar o modelo de conhecimento especializado de professores de física (PTSK). Congresso Internacional de Formação e Desenvolvimento Profissional Docente - Residência Docente: Paradigma De Integração Teoria-Prática, 3., Cuiabá. Anais... p. 1 - 5.

Lopes, A. R. C. (1997) Conhecimento Escolar em Química - Processo de Mediação Didática da Ciência. Química Nova na Escola, SBQ, 5.

Loughran, J. et al. (2001) Documenting science teachers' pedagogical content knowledge through PaP-eRs. Research in Science Education, 31(2), $289-307$.

Luis, M. (2021) O Conhecimento Especializado do Professor quando ensina Tópicos de Biologia. Tese (Doutorado). Universidad de Huelva - UHU, Huelva, $234 \mathrm{p}$.

Luís, M., Monteiro, R. \& Carrillo, J. (2015) Conhecimento Especializado do Professor para Ensinar Ciências. In: Encontro Nacional de Educação em Ciências, XVI., Lisboa, Portugal. Anais... APEduC, 1, 1 - 6.

Marques, M.\& Moriel Junior, J. G. (2019) Conhecimento especializado de professores de biologia: uma análise de PaP-eR sobre embriologia humana. In: $I V$ Congreso Iberoamericano Sobre Conocimiento Especializado del Profesor de Matemáticas, Huelva: Universidad de Huelva Publicaciones. Anais... p.127134.

Marques, M. (2020) Conhecimento especializado de professores de biologia: análise de relatos de prática no Ensino Médio. Dissertação (Mestrado em Ensino) - Programa de Pós-Graduação Stricto Sensu em Ensino, Instituto Federal de Educação, Ciência e Tecnologia de Mato Grosso - IFMT, Cuiabá, 110f.

Marques, M. \& Moriel Junior. (2020) Conhecimentos Especializados de Professor de Biologia Mobilizados em uma Aula Prática Sobre Interações Ecológicas. Revista REAMEC, 8(2), 253-271.

Marques, M., Soares, S. T. C. \& Moriel Junior, J. G. (2021) Conhecimentos especializados mobilizados em uma aula prática de biologia sobre sistema respiratório. Revista Multidisciplinar.com, 3(1), 81-100.

Martins, J. E. A. (2021) Conhecimento especializado de professores de química (CTSK): estudo de uma experiência de ensino sobre hidrocarbonetos. Dissertação (Mestrado em Ensino) - Programa de Pós-Graduação Stricto Sensu em Ensino, Instituto Federal de Educação, Ciência e Tecnologia de Mato Grosso - IFMT, Cuiabá. 96f.

Martins, J. E., Carbo, L., Soares, S. T. C. (2021) Conhecimento especializado de professores de química - CTSK: uma análise de prática docente no ensino de hidrocarbonetos. Revista Prática Docente, 6(1), 1-23.

Moriel Junior, J. G. (2014) Conhecimento especializado para ensinar divisão de frações. Tese (Doutorado em Educação em Ciências e Matemática) Programa de Pós-Graduação em Educação em Ciências e Matemática - PPGECEM, Rede Amazônica de Educação em Ciências e Matemática - REAMEC, Universidade Federal de Mato Grosso - UFMT, Cuiabá. 162 p.

Moriel Junior, J. G. \& Wielewski, G. D. (2017) Base de Conhecimento de Professores de Matemática: do Genérico ao Especializado. Rev. Ens. Educ. Cienc. Human., 18(2), 126-133.

Oliveira, I \& Rezende, F. (2011) Discurso de Estudantes e Habitus Pedagógico em Cursos de Graduação em Ciências Naturais. Revista Brasileira de Pesquisa em Educação em Ciências - RBPEC, UFMG. 11(3).

Pazinato, M. S., Braibante, H. T. S., Braibante, M. E. F., Trevisan, M. C., Silva, G. S. (2012) Uma Abordagem Diferenciada para o Ensino de Funções Orgânicas através da Temática Medicamentos. Química Nova na Escola, SBQ, 34(1), 21-25.

Quadros, A. L, Silva, D. C., Andrade, F. P, Aleme, H. G, Rodrigues, S. O, Silva, G. F. (2011) Ensinar e aprender Química: a percepção dos professores do Ensino Médio. Educar em Revista, UFPR, Curitiba, n. 40, p. 159-176.

Shulman, L. S. Artigos. Professor of Education Emeritus, Stanford University, California, USA. <https://scholar.google.com.br/citations?user=QRWVLcAAAAJ\&hl=pt-BR>

Shulman, L. S. (1986) Those who understand: Knowledge growth in teaching. Education Researcher, SAGE, California, USA, 4-14.

Silva, R. M. G \& Schnetzler, R. P. (2005) Constituição de professores universitários de disciplinas sobre ensino de Química. Química Nova, SBQ, São Paulo 28(6).

Soares, S. T. C. (2019) Conhecimento Especializado de Professores de Química - CTSK: Proposta de Modelo Teórico. Dissertação (Mestrado em Ensino) Programa de Pós-Graduação Stricto Sensu em Ensino, Instituto Federal de Educação, Ciência e Tecnologia de Mato Grosso (IFMT), Cuiabá. 113f.

Soares, S. T. C., Lima, S. S. \& Carbo, L. (2020) Conhecimento especializado de professores de química: modelo teórico. Revista REAMEC, 8(2), 648-666.

Vasco, D., Moriel Junior, J. \& Contreras, L. C. (2017) Subdominios del mathematics teacher's specialised knowledge (MTSK): KoT y KSM: definición, categorías y ejemplos. Jornadas de Investigación em Didática de las Matemáticas, 3., 2017, Huelva, Espanha. p. 29-37. 